\title{
GLOBAL SOLUTIONS OF HYPERBOLIC SYSTEMS OF CONSERVATION LAWS IN TWO DEPENDENT VARIABLES
}

\author{
BY J. L. JOHNSON AND J. A. SMOLLER ${ }^{1}$
}

Communicated by J. K. Moser, March 28, 1968

We are interested in general hyperbolic systems of the form

$$
u_{t}+f(u, v)_{x}=0, \quad v_{t}+g(u, v)_{x}=0
$$

with initial data

$$
(v(0, x), u(0, x))=\left(v_{0}(x), u_{0}(x)\right) .
$$

The vector $U=(v, u)$ is a function of $t$ and $x, t \geqq 0,-\infty<x<\infty$, and the functions $f$ and $g$ are $C^{2}$ functions of two real variables. We assume that the system (1) is hyperbolic in some open set $u$ in the $v-u$ plane, with $f_{v} g_{u}>0$. Let $D F(U)$ and $D^{2} F(U)$ denote respectively the first and second Fréchet derivatives (see [2]) of the vector function $F=(f, g): \mathcal{U} \rightarrow R^{2}$; and let $r_{j}(U), j=1,2$, be the eigenvectors of $D F(U)$, with orthogonal vectors $l_{j}(U), j=1,2: l_{i}(U) r_{j}(U)=0$ for $i \neq j$.

THEOREM 1. Let the system (1) be hyperbolic in an open set $\mathfrak{u}$ in the $v-u$ plane. Then (a) the system (1) is genuinely nonlinear in the jth characteristic field at $U \in \mathcal{U}$ (see Lax [6]) if and only if

$$
l_{j}(U) D^{2} F(U)\left[r_{j}(U), r_{j}(U)\right] \neq 0 ;
$$

(b) the system (1) satisfies the Glimm-Lax shock interaction condition (condition (c) of [4]) in $u$ provided that left eigenvectors $l_{j}(U)$ can be chosen so that

$$
l_{j}(U) D^{2} F(U)\left[r_{k}(U), r_{k}(U)\right]>0, \quad j, k=1,2, j \neq k, U \in \mathcal{u} .
$$

The Glimm-Lax shock interaction condition states that the interaction of two shocks of one family produces a shock of the same family and a rarefaction wave of the opposite family. Moreover, for sufficiently weak shocks, we are able to prove an analogous theorem for $n \times n$ systems of conservation laws, $n \geqq 2$, which locally admit Riemann invariants. The proof of it uses some ideas in [3].

We assume that the system (1) is genuinely nonlinear in $\mathcal{U}$, and we normalize $r_{j}$ by $D \lambda_{j}(U)\left[r_{j}(U)\right]>0, j=1,2$, where $\lambda_{j}=\lambda_{j}(U)$ is the eigenvalue asociated with $r_{j}, \lambda_{2}>\lambda_{1}$. We then normalize $l_{j}$ by $l_{j} r_{j}>0$,

${ }^{1}$ Research supported by NSF research contract No. GP-7445. 
$j=1,2$. Our additional assumption on the system (1) is that $l_{j}(U) D^{2} F(U)\left[r_{k}(U), r_{k}(U)\right]>0, \quad j, k=1,2, U \in \mathcal{u}$.

We define ${ }^{2}$ a shock wave of the $i$ th characteristic field $i=1,2$, to be a discontinuity $x=x(t)$ satisfying the Rankine-Hugoniot condition and the inequality

$$
\lambda_{i}(U(x+0, t))<\dot{x}(t)<\lambda_{i}(U(x-0, t)) .
$$

Theorem 2. For each point $P_{0}=\left(v_{0}, u_{0}\right)$ in $u$, there exist two smooth curves through $P_{0}, u=s\left(v ; P_{0}\right)$ and $u=w\left(v ; P_{0}\right)$, called the shock and wave curves respectively, defined in $u$ globally, which consist of states that can be connected to $P_{0}$ by a shock wave of the second characteristic field, and a rarefaction wave of the first characteristic field, respectively.

For each $P_{0}=\left(v_{0}, u_{0}\right)$ in $\mathcal{U}$, we require that

$$
\sigma\left(v, u ; v_{0}, u_{0}\right)>\lambda_{1}\left(v_{0}, u_{0}\right)
$$

for all $(v, u) \in \mathcal{u}$ with $u=s\left(v ; P_{0}\right)$, where $\sigma\left(v, u ; v_{0}, u_{0}\right)$ is the corresponding shock speed. This requirement is satisfied for example, if any of the following conditions hold in $\mathcal{U}$ :

(a) $\lambda_{2} \geqq 0 \geqq \lambda_{1}$,

(b) $\partial \lambda_{1} / \partial u \leqq 0$,

(c) $f_{u v} \geqq 0$ and $f_{u u} \leqq 0$ or $g_{v u} \geqq 0$ and $g_{v v} \leqq 0$.

Fix a point $P_{0}=\left(v_{0}, u_{0}\right)$ in the $v-u$ plane and let

$$
C\left(P_{0}\right)=\left\{(v, u) \in \mathcal{u}: v \geqq v_{0}, s\left(v ; P_{0}\right) \leqq u \leqq w\left(v ; P_{0}\right)\right\} .
$$

These regions $C(P)$ then satisfy the following order condition.

Theorem 3. If $P_{1} \in C\left(P_{0}\right)$, then $C\left(P_{1}\right) \subseteq C\left(P_{0}\right)$.

To prove this theorem, we first consider the case where $P_{1}=\left(v_{1}, u_{1}\right)$ lies on the shock curve starting at $P_{0} ;$ i.e., $P_{1}$ satisfies $u_{1}=s\left(v_{1} ; P_{0}\right)$. If $u_{2}=s\left(v_{2} ; P_{1}\right)$ is any point on the shock curve from $P_{1}$, then we shall show that $\left(v_{2}, u_{2}\right)$ is not on the shock curve from $P_{0}$; i.e., we shall show that $u_{2} \neq s\left(v_{2} ; P_{0}\right)$. Suppose that this is not the case and let $\sigma_{01}, \sigma_{02}$, $\sigma_{12}$ be the corresponding shock speeds. Then

$$
\begin{aligned}
& \sigma_{01}\left(P_{1}-P_{0}\right)=F\left(P_{1}\right)-F\left(P_{0}\right), \\
& \sigma_{12}\left(P_{2}-P_{1}\right)=F\left(P_{2}\right)-F\left(P_{1}\right), \\
& \sigma_{02}\left(P_{2}-P_{0}\right)=F\left(P_{2}\right)-F\left(P_{0}\right) .
\end{aligned}
$$

Adding the first two equations and comparing with the third shows that

2 Note that this definition differs slightly from the definition in [6]. 


$$
\sigma_{01}\left(P_{1}-P_{0}\right)+\sigma_{12}\left(P_{2}-P_{1}\right)=\sigma_{02}\left(P_{2}-P_{0}\right)=\sigma_{02}\left(P_{2}-P_{1}\right)+\sigma_{02}\left(P_{1}-P_{0}\right) .
$$

If the vectors $P_{1}-P_{0}$ and $P_{2}-P_{1}$ were not collinear, we would have $\sigma_{01}=\sigma_{02}=\sigma_{12}$ in contradiction to the shock condition $\sigma_{01}>\lambda_{2}\left(P_{1}\right)>\sigma_{12}$. Hence we conclude that these vectors are collinear so that

$$
\left(u_{1}-u_{0}\right) /\left(v_{1}-v_{0}\right)=\left(u_{2}-u_{1}\right) /\left(v_{2}-v_{1}\right)=\left(u_{2}-u_{0}\right) /\left(v_{2}-v_{0}\right) .
$$

But this too is impossible since we can easily show that the derivative of $\left(u-u_{0}\right) /\left(v-v_{0}\right)$ along the shock curve $u=s\left(v ; P_{0}\right)$ is positive; i.e., that the shock curve is convex. (We remark that this part of the theorem is proved without using condition (3), and shows that in $\mathfrak{u}$, the interaction of two shocks of the same family produces a shock of the same family plus a rarefaction wave of the opposite family.) For the general case, we first show that the theorem holds if and only if for each $P_{1}=\left(v_{1}, w\left(v_{1} ; P_{0}\right)\right)$ with $v_{1}>v_{0}, u=s\left(v ; P_{1}\right)$ implies $u \geqq s\left(v ; P_{0}\right)$; i.e., the theorem holds if and only if for every point $P_{1}$ on the wave curve through $P_{0}$, the shock curve starting at $P_{1}$ does not go below the shock curve starting at $P_{0}$. We then show that condition (3) implies (actually is equivalent to) this latter condition. We remark that Theorem 3 holds if instead of assuming condition (3), we have a uniqueness theorem for Riemann problems in $C\left(P_{0}\right)$. Hence the theorem will hold, for example, if instead of condition (3), the conditions for uniqueness of "decay of a discontinuity" as described in [7] are satisfied in $\mathcal{~}$. Thus (3) is a necessary condition if (1) has a unique solution to the Cauchy problem.

In order to prove a global existence theorem for the problem (1), (2), we assume that the initial data satisfies a certain order condition which we now describe. Suppose that the "curve" $u=u_{0}(x), v=v_{0}(x)$, $-\infty<x<\infty$, is bounded and contained in $\mathcal{u}$. Our order condition states that if we let $\left(v_{i}, u_{i}\right), i=1,2$ be two points on this curve corresponding to the points $x_{i}, i=1,2$ respectively, where $x_{1}<x_{2}$, then the Riemann problem for (1) with initial data

$$
\begin{aligned}
\left(v_{0}(x), u_{0}(x)\right) & =\left(v_{1}, u_{1}\right), & & x<0, \\
& =\left(v_{2}, u_{2}\right), & & x>0,
\end{aligned}
$$

is resolved in $\mathfrak{U}$ by a 2 -shock and a 1-rarefaction wave. Under these hypotheses we can prove

Theorem 4. The Cauchy problem (1), (2) has a global solution contained in $\mathrm{u}$.

(Similar theorems can be proved in the case where the data is resolved in $u$ by a 1 -shock and a 2 -rarefaction wave.) 
Theorems 2, 3 and 4 are extensions of similar theorems found in [5] and [8] where the cases $f_{u}=g_{v}=0$ and $f_{u}=g_{v}=g_{u u}=0$ respectively, are considered, and $\mathcal{U}$ is the half-space $v>0$. We prove these theorems by extending and simplifying the methods in [5]. In the proof of theorem 4, we find a solution of (1), (2) as a limit of a sequence of solutions of (1) with step data. We show that these approximating solutions are uniformly bounded and have uniformly bounded variation locally in the sense of Tonelli-Cesari, [1], with respect to two independent (not necessarily orthogonal) directions. It then follows that this sequence is compact in the topology of $L_{1}$-convergence on compacta, and therefore a subsequence converges to a solution of the problem (1), (2).

In addition to these theorems, we have proved existence theorems for the problems (1), (2) with the same hypotheses on $f, g$ and the initial data, using the difference scheme introduced by Glimm in [3]. Thus the Glimm scheme can be used to solve certain initial-value problems where the variation of the initial data is arbitrarily large.

The complete proofs of these results will appear elsewhere.

\section{REFERENCES}

1. L. Cesari, Sulle funzioni a variazione limita, Ann. Scuola Norm. Pisa (2) 5 (1936), 299-313. 1960.

2. J. Dieudonne, Foundations of modern analysis, Academic Press, New York,

3. J. Glimm, Solutions in the large for nonlinear hyperbolic systems of equations, Comm. Pure Appl. Math. 18 (1965), 697-715.

4. J. Glimm and P. D. Lax, Decay of solutions of systems of hyperbolic conservation laws, Bull. Amer. Math. Soc. 73 (1967), 105.

5. J. L. Johnson and J. A. Smoller, Global solutions of certain hyperbolic systems of quasi-linear equations, J. Math. Mech. 17 (1967), 561-576.

6. P. D. Lax, Hyperbolic systems of conservation laws. II, Comm. Pure Appl. Math. 10 (1957), 537-566.

7. B. L. Rozdestvenskii, Discontinuous solutions of systems of quasilinear hyperbolic equations, Uspehi Mat. Nauk 15 (1960), no. 6 (96), 59-117= Russian Math. Surveys 15 (1960), no. 6, 53-111.

8. Zhang Tong and Guo Yu-Fa, $A$ class of initial-value problems for systems of aerodynamic equations, Acta. Math. Sinica 15 (1965), 386-396=Chinese Math. Acta 7 (1965), 90-101.

Naval Electronics laboratory Center for Command Control and Communications, SAN Diego, California and

The University of Michigan 